\title{
IETF Reliable and Available Wireless (RAW): Use Cases and Problem Statement
}

\author{
Georgios Z. Papadopoulos ${ }^{1[0000-0002-0331-0579]}$, Fabrice \\ Theoleyre $^{2[0000-0002-7903-3520]}$, Pascal Thubert ${ }^{3[0000-0002-1615-1650]}$, and \\ Nicolas Montavont ${ }^{1}$ \\ 1 IMT Atlantique, Irisa, France \\ firstname. lastname@imt-atlantique.fr \\ 2 University of Strasbourg / CNRS, ICube Lab, France \\ theoleyre@unistra.fr \\ 3 Cisco Systems, France \\ pthubert@cisco.com
}

\begin{abstract}
Due to uncontrolled interferences, including the self-induced multi-path fading, deterministic networking is difficult to achieve on wireless links. The radio conditions may change much faster than a centralized routing paradigm can adapt and reprogram, in particular when the controller is distant and connectivity is slow and limited. Reliable and Available Wireless (RAW) separates the routing time scale at which a complex path is recomputed from the forwarding time scale at which the forwarding decision is taken for an individual packet. RAW operates at the forwarded time scale. The RAW problem is to decide, within the redundant solutions that are proposed by the routing plane, which will be used for each individual packet to provide a Deterministic Networking (DetNet) service while minimizing the waste of resources. A solution would consist of a set of protocols that evaluate the media in real time and another that controls the use of redundancy and diversity attributes that are available along the path. In this paper, we first introduce the motivation behind this approach along with the industrial use cases that requires RAW characteristics. We then give an overview of the ongoing related works at the Internet Engineering Task Force (IETF). Finally, we present the RAW problem statement.
\end{abstract}

Keywords: Reliable and Available Wireless · RAW - DetNet · LLNs . PAREO Functions · Industrial Wireless Networks.

\section{Introduction}

Wireless networks operate on a shared medium where uncontrolled interference, including the self-induced multi-path fading, adds another dimension to the statistical effects that affect the packet delivery. Scheduling transmissions can alleviate those effects by leveraging diversity in the spatial, time, code, and frequency domains, and provide a Reliable and Available service while preserving energy, and optimizing the use of the shared spectrum. 
Bringing determinism in a packet network means eliminating the statistical effects of multiplexing that result in probabilistic jitter and loss. This can be approached with a tight control of the physical resources to maintain the amount of traffic within a limited volume of data per unit of time that fits the physical capabilities of the underlying technology, and the use of time-shared resources (bandwidth and buffers) per circuit, and/or by shaping and/or scheduling the packets at every hop.

Deterministic Networking (DetNet) is an attempt to mostly eliminate packet loss for a committed bandwidth with a guaranteed worst-case end-to-end latency, even when co-existing with best-effort traffic in a shared network. This innovation is enabled by recent developments in technologies including IEEE 802.1 TSN (for Ethernet LANs) and IETF DetNet (for wired IP networks). It is getting traction in various industries including manufacturing, online gaming, professional $\mathrm{A} / \mathrm{V}$, cellular radio and others, making possible many cost and performance optimizations. The DetNet architecture [9] is composed of three planes: a (User) Application Plane, a Controller Plane, and a Network Plane. Reliable and Available Wireless (RAW) [6] extends DetNet to focus on issues that are mostly a concern on wireless links, and inherits the architecture and the planes. A RAW Network Plane is thus a Network Plane inherited by RAW from DetNet.

RAW networking aims at providing highly available and reliable end-to-end performances in a network with scheduled wireless segments. Uncontrolled interference and transmission obstacles may impede the transmission, while techniques such as beamforming with Multi-user MIMO (MU-MIMO) can only alleviate some of those issues. This uncertainty places limits on the computation of the amount of traffic that can be transmitted on a link while conforming to a RAW Service Level Agreement (SLA) that may vary rapidly. Moreover, the wireless and wired media are fundamentally different at the physical level, and the methods to achieve RAW will differ from those used to support time-sensitive networking over wires, as a RAW solution will need to address less consistent transmissions, energy conservation and shared spectrum efficiency. However, recent efforts at the Institute of Electrical and Electronics Engineers (IEEE) and 3GPP indicate that wireless is finally catching up at the lower layer and that it is now possible for the Internet Engineering Task Force (IETF) to extend DetNet for wireless segments that are capable of scheduled wireless transmissions.

The intent for RAW is to provide DetNet elements that are specialized for short range radios. From this inheritance, RAW stays agnostic to the radio layer underneath though the capability to schedule transmissions is assumed. How the PHY is programmed to do so, and whether the radio is single-hop or meshed, are hidden for the IP layer and are not part of the RAW abstraction. Still, in order to focus on real-world issues and assert the feasibility of the proposed capabilities, RAW will focus on selected technologies that can be scheduled at the lower layers: IEEE Std. 802.15.4 Time-Slotted Channel Hopping (TSCH), 3GPP 5G Ultra-Reliable Low Latency Communications (URLLC), IEEE 802.11ax/be 
where 802.11be is Extreme High Throughput (EHT), and L-band Digital Aeronautical Communications System (LDACS) [26].

The establishment of a path is not in-scope for RAW. It may be the product of a centralized Controller Plane as described for DetNet. As opposed to wired networks, the action of installing a path over a set of wireless links may be very slow relative to the speed at which the radio conditions vary. Moreover, it makes sense in the wireless case to provide redundant forwarding opportunities at the IP level. RAW distinguishes the longer time scale at which routes are computed from the the shorter forwarding time scale where per-packet decisions are made. RAW operates at the forwarding time scale on one flow over one path that is preestablished and installed by means of techniques which are outside of the scope of RAW. The scope of the RAW Working Group (WG) comprises Network plane protocol elements such as Operations, Administration, and Maintenance (OAM) and in-band control to improve the RAW operation at the Service and at the forwarding sub-layers, e.g., controlling whether to use packet replication, Hybrid ARQ and coding, with a constraint to limit the use of redundancy when it is really needed, e.g., when a spike of loss is observed. This is discussed in more details in Section 4 and the next sections.

This paper is organized as follows. In Section 2, we present a number of wireless use cases that demonstrate the need for RAW capabilities. Then, section 3 exposes the related work that has been done at the IETF. We describe in Section 4 the terminology that RAW uses. In Section 5, we detail the RAW approach, describing first the difference between the routing and forwarding time scales, and then exposing the RAW problem statement, i.e., the WG charter. Finally, Section 6 concludes the paper.

\section{Use Cases}

Many Industrial Applications rely on a deterministic industrial network [11]. However, enabling wireless communications also multiplies the possibilities [17]. We detail here a few use cases that may benefit from a RAW solution.

\subsection{Industry 4.0 and Robotics}

Industry 4.0 represents the next industrial revolution [15]. The objective consists in collecting a huge volume of data in real-time to adapt dynamically the industrial process. Thus, we need big data tools to handle the huge volume of data, and to extract interesting features.

However, to enable Cyber Physical Systems (CPS), we need also a network infrastructure able to forward critical flows, guaranteeing both ultra reliability and small and bounded end-to-end latencies. The DetNet working group has provided pioneering piece of work to standardize the communication stack, so that resources can be allocated end-to-end [10]. It relies mostly on the IEEE 802.1 Time Sensitive Networking (TSN) features [29]. Each switch on the path is able to pre-reserve a port to a specific flow: periodically, a flow has the full 


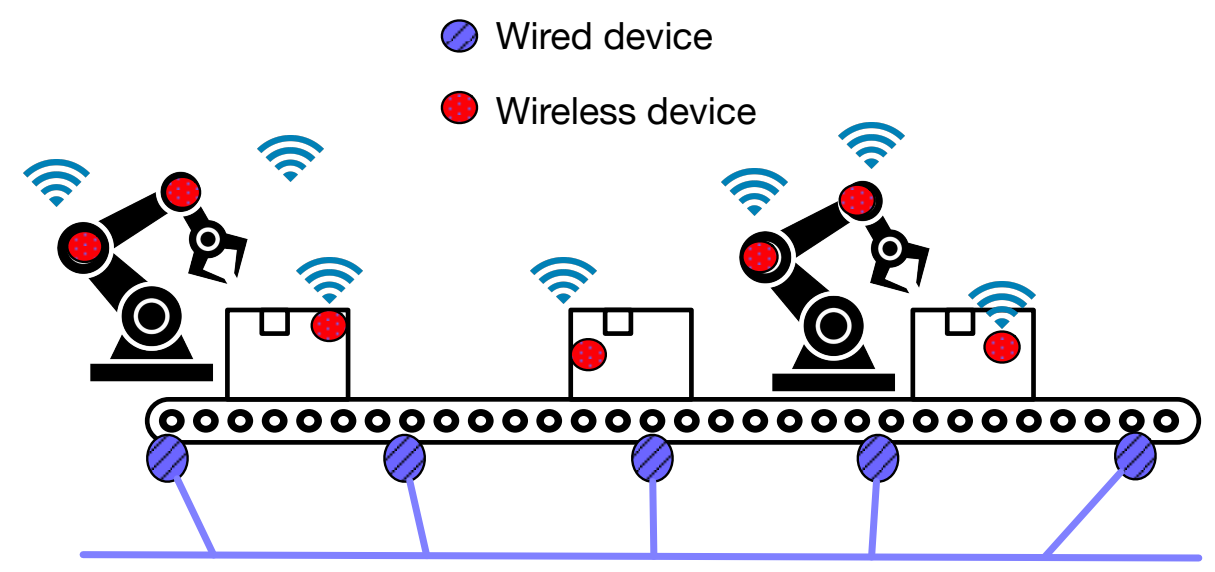

Fig. 1. Wireless Industrial Networks.

priority to send its packets through a physical port. This way, TSN removes the competition, and thus transforms the Ethernet network into a fully deterministic infrastructure. While industrial networks are expected to integrate wireless communications in the future, wireless transmissions are known to be time-variant, and lossy [20], [18], [19]. In these conditions, providing ultra high reliability, and a low latency is particularly challenging.

A typical use case mixes both wired and wireless parts (Fig. 1). While some sensors on the manufacturing line may be directly connected to the wired infrastructure using e.g., DetNet, some wireless devices are also present. For instance, robotic arms are mobile and wires tend to cause premature wear and malfunctions. In such infrastructure cohabit very different flows: a geofencing application with an accuracy of a few seconds, or robots with almost real time control. For instance, human and robotic safe integration needs 15 to $36 \mathrm{~ms}$ end-to-end latencies [22].

\subsection{Gaming and Multimedia}

Current home consoles consist of a single computational device, and a few wireless remote controls. However, we face to increasingly complex interactions, that integrate virtual reality devices, smartphones, other home consoles, headsets, etc. [14].

While some displays and the gaming server may be connected through a high bandwidth network (Fig. 2), some devices need wireless communications. Remote control and virtual reality headsets typically cannot function properly with cables since they would negatively impact the user experience. Moreover, multi camera techniques for motion capture [8] generate a huge volume of data to process in real-time. Last but not least, multiple wireless technologies may cohabit to fit the different requirements (motion capture vs. remote controls). A 


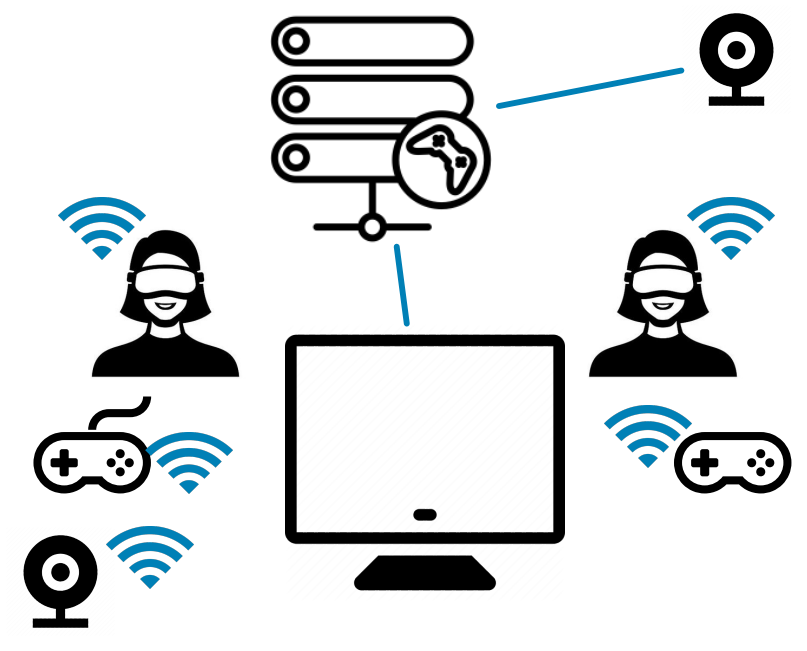

Fig. 2. Home gaming scenario.

unified way to manage the wireless infrastructure is required to allocate properly the radio resources, and to limit co-interferences.

Artistic applications that exploit gaming technologies have started to emerge. By creating installations that react to the audience, modern art tends to now abolish the frontiers between the real and digital, imaginative worlds. In particular, digital fulldomes provide a full 360 experience [23], where the digital worlds may react dynamically to the actions of the audience and the artists. For this purpose, we need wireless communications with a very small latency to provide natural interactions. Digital walls would represent also a promising futuristic Human-Computer Interface [12], using partly wireless devices to personalize the user experience. Using wireless part allow a more flexible reconfiguration, assembling the different blocks very easily.

\subsection{Smart Building and Home Automation}

Smart buildings pave the way for a reduction of the carbon footprint, and for a better usage of offices and homes. In particular, energy management seems a key enabler: the objective is to dynamically adapt the heating or HVAC system to the building occupancy [7]. A collection of sensors detect the presence of occupants, or may even identify uniquely each individual bodies to adapt the behavior of the room to the users. To maximize the reconfigurability, most sensors and actuators may use wireless transmissions. Moreover, deploying kilometers of cables in existing buildings seems unreasonable, and a clean slate approach is unrealistic as well.

Many flows may cohabit in the same infrastructure with very different characteristics. Temperature sensors generate small packets periodically, every e.g., 


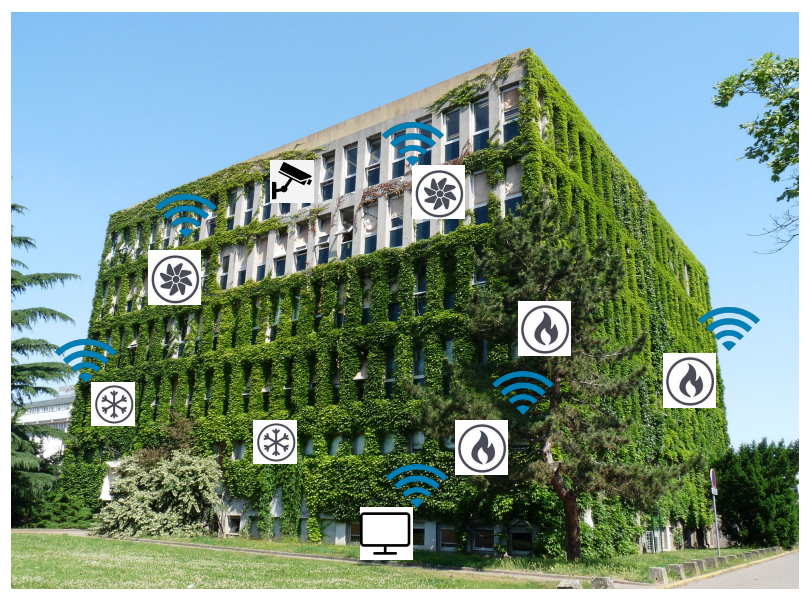

Fig. 3. Smart building scenario.

minute, while Closed-Circuit TeleVision (CCTV) generate multimedia streams toward the video servers. Smart buildings require to interconnect the different applications (cf. Fig. 3), so that the different sensors send their data to multiple controllers, to make smart decisions. Thus, we need a global solution able to jointly optimize all the transmissions, with different co-interfering wireless technologies.

\section{Related Work at The IETF}

RAW intersects with existing protocols or practices in development at the IETF. In particular, the Dynamic Link Exchange Protocol (DLEP) [21] from Mobile Ad Hoc Networks (MANET) [1] can be leveraged at each hop to derive generic radio metrics (e.g., based on Link Quality Indicator (LQI), Received Signal Strength Indicator (RSSI), queueing delays and Expected Transmission Counts (ETX)) on individual hops. These metrics are typically useful for RAW since the radio link quality is time-variant in radio networks, and have to be carefully estimated to size correctly the amount of bandwidth to reserve for a flow.

In the same way, OAM for DetNet [16] allows to observe the state of the IP Data Plane. Typically, it monitors MultiProtocol Label Switching (MPLS) and IPv6 pseudowires [28], in the direction of the traffic. RAW needs feedback that flows on the reverse path and gathers instantaneous values from the radio receivers at each hop to inform back the source and replicating relays so they can make optimized forwarding decisions.

In RAW, we need also to be fault tolerant. In particular, Bidirectional Forwarding Detection (BFD) [2] detects faults in the path between an ingress and an egress forwarding engines, but is unaware of the complexity of a path with 
replication, and expects bidirectionality. BFD considers delivery as success while RAW has also to consider the end-to-end latency.

SPRING [5] and BIER [3] define in-band signaling that influences the routing when decided at the head-end on the path. A draft [27] already addresses this problem, and more may follow. RAW will need new in-band signaling when the decision is distributed, e.g., required chances of reliable delivery to destination within latency. This signaling enables relays to tune retries and replication to meet the required SLA.

The Common Control and Measurement Plane (CCAMP) [4] defines protocolindependent metrics and parameters (measurement attributes) for describing links and paths that are required for routing and signaling in technology-specific networks. RAW would be a source of requirements for CCAMP to define metrics that are significant to the radios networks.

\section{Definitions}

RAW defines the following terms:

PAREO (Packet Automatic Repeat reQuest, Replication and Elimination, and Ordering (PAREO)) [13] is a superset of DetNet's Packet Replication, Elimination, and Ordering Functions (PREOF) that includes radio-specific techniques such as short range broadcast, MU-MIMO, constructive interference and overhearing, which can be leveraged separately or combined to increase the reliability.

Flapping corresponds to a radio link with a very time-variant quality. Typically, the wireless connectivity drops abruptly for a short period of time, for e.g., a few hundreds of milliseconds, or even a few seconds.

Reliability measures the probability that an item will perform its intended function for a specified interval under stated conditions. For RAW, the service that is expected is delivery within a bounded latency and a failure is when the packet is either lost or delivered too late. RAW expresses reliability in terms of Mean Time Between Failure (MTBF) and Maximum Consecutive Failures (MCF).

Availability measures the relative amount of time where a path operates in stated condition, in other words (uptime)/(uptime+downtime). Because a serial wireless path may not be good enough to provide the required availability, and even two parallel paths may not be over a longer period of time, the RAW availability implies a path that is a lot more complex than what DetNet typically envisages (a Track).

a Track abstracts the underlaying technology, and represents a set of resources along the path from the source to the destination. RAW specifies strict and loose Tracks depending on whether the path is fully controlled by RAW or traverses an opaque network where RAW cannot observe and control the individual hops. 


\section{RAW approach}

A prerequisite to the RAW work is that an end-to-end routing function computes a complex sub-topology along which forwarding can happen between a source and one or more destinations. This represents a Track, as specified in the $6 \mathrm{TiSCH}$ Architecture [25]. Tracks provide a high degree of redundancy and diversity and enable DetNet PREOF, end-to-end network coding, and possibly radiospecific abstracted techniques such as ARQ, overhearing, frequency diversity, time slotting, and possibly others.

How the routing operation computes the Track is out of scope for RAW. The scope of the RAW operation is one Track, and the goal of the RAW operation is to optimize the use of the Track at the forwarding timescale to maintain the expected service while optimizing the usage of constrained resources such as energy and spectrum.

Another prerequisite is that an IP link can be established over the radio with some guarantees in terms of service reliability, e.g., it can be relied upon to transmit a packet within a bounded latency and provides a guaranteed BER/PDR outside rare but existing transient outage windows that can last from split seconds to minutes. The radio layer can be programmed with abstract parameters, and can return an abstract view of the state of the Link to help forwarding decision (think Dynamic Link Exchange Protocol (DLEP) from MANET). In the layered approach, how the radio manages its PHY layer is out of control and out of scope. Whether it is single hop or meshed is also unknown and out of scope.

\subsection{Routing Time Scale vs. Forwarding Time Scale}

With DetNet, the end-to-end routing can be centralized and can reside outside the network. In wireless, and in particular in a wireless mesh, the path to the controller is expensive to maintain, consuming both air time and energy. Reaching the routing steady state can also be slow in regards to the speed of events that affect the forwarding operation at the radio layer. Due to the cost and latency to perform a route computation, the controller plane is not expected to be sensitive/reactive to transient changes. The abstraction of a link at the routing level is expected to use statistical operational metrics that aggregate the behavior of a link over long periods of time, and represent its availability as shades of gray as opposed to either up or down.

In the case of wireless, the changes that affect the forwarding decision can happen frequently and often for short durations, e.g., a mobile object moves between a transmitter and a receiver, and will cancel the line of sight transmission for a few seconds, or a radar measures the depth of a pool and interferes on a particular channel for a split second, see Fig. 4.

There is thus a desire to separate the long term computation of the route and the short term forwarding decision. In such a model, the routing operation computes a complex Track that enables multiple Non-Equal Cost Multi-Path (N-ECMP) forwarding solutions, and leaves it to the forwarding plane to make the per-packet decision of which of these possibilities should be used. 


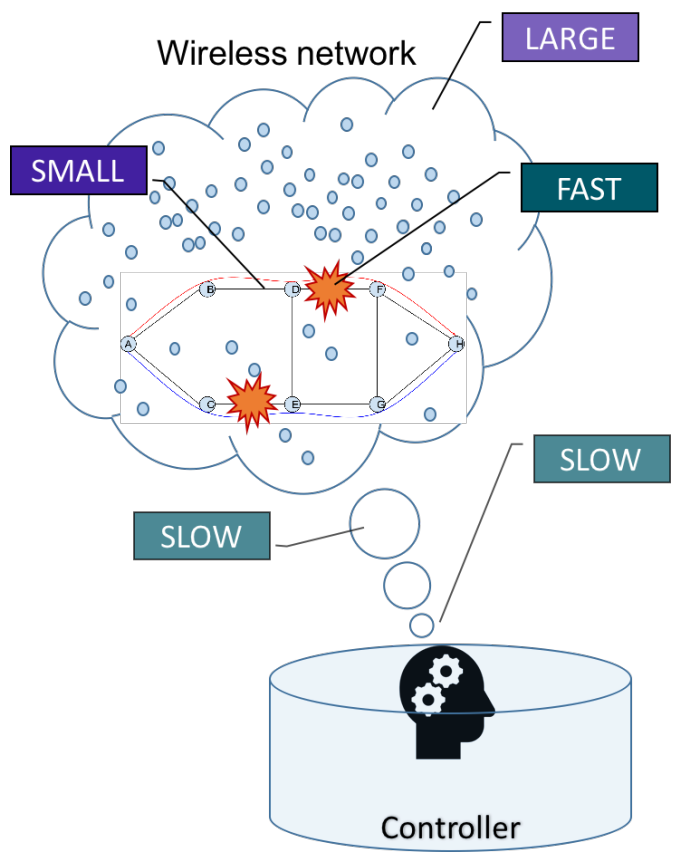

Fig. 4. A multi-hop RAW network that may represent potential delays on routing and forwarding decisions due to the network instabilities.

This concept is already widely used in wired networks, where traffic engineering exploits multiple alternate paths, using e.g. OAM in MPLS-TP or BFD over a collection of SD-WAN tunnels. RAW brings this concept in the wireless world, where the forwarding time scale is an order(s) of magnitude shorter than the controller plane routing time scale. It separates the protocols and metrics that are used at both scales. Routing can operate on long term statistics such as delivery ratio over minutes to hours, but as a first approximation can ignore flapping. On the other hand, the RAW forwarding decision is made at packet speed, and uses information that must be pertinent at the present time for the current transmission.

\subsection{Problem Statement}

Within a large routed topology, the routing operation builds a particular complex Track with one source and one or more destinations; within the Track, packets may follow different paths and may be subject to RAW forwarding operations that include replication, elimination, retries, overhearing and reordering.

The RAW forwarding decisions include the selection of points of replication and elimination, how many retries can take place, and a limit of validity for 
the packet beyond which the packet should be destroyed rather than forwarded uselessly further down the Track.

The decision to apply the RAW techniques must be done quickly, and depends on a very recent and precise knowledge of the forwarding conditions within the complex Track. There is a need for an observation method to provide the RAW forwarding plane with the specific knowledge of the state of the Track for the type of flow of interest (e.g., for a QoS level of interest). To observe the whole Track in quasi real time, RAW will consider existing tools such as L2-triggers, DLEP, BFD and in-band and out-of-band OAM [24].

RAW forwarding decisions may be implemented at the ingress and piggybacked, in-band, in the packet, which requires new loose or strict Hop-by-hop signaling. To control the RAW forwarding operation along a Track for the individual packets, RAW may leverage and extend known techniques such as DetNet tagging, Segment Routing (SRv6) or BIER-TE such as done with BIERPREF [27].

An alternate way is to enable each forwarding node to make the RAW forwarding decisions for a packet on its own, based on its knowledge of the expectation (timeliness and reliability) for that packet and a recent observation of the rest of the way across the possible paths within the Track. Information about the service should be placed in the packet and matched with the forwarding node's capabilities and policies. In either case, a per-flow state is installed in all intermediate nodes to recognize the flow and determine the forwarding policy to be applied.

\section{Conclusions}

Industrial wireless networks promise to enable reconfigurability while still providing strict guarantees concerning the reliability and the end-to-end delay. We presented several use cases, that may benefit from a wireless infrastructure, to send even critical flows. We presented here the RAW working group, that aims to design the network protocols able to exploit multiple wireless technologies. Since radio links are known to be lossy, RAW proposes to separate the routing (long) timescale from the forwarding (short) timescale. While RAW is in charge of constructing end-to-end redundant paths to cope with packet losses, the lower layers are in charge of taking the forwarding decisions, so that e.g., temporary link flapping may be combatted.

In the future, we expect to design the routing algorithms able to construct accurately the different paths (independency, complementary, load-balancing), adapted for the wireless forwarding plane. We also plan to explore how existing OAM tools are adapted for wireless networks, and to propose protocols and algorithms to cope with their specificities, for instance, to detect flapping links, or temporary and permanent route breaks. 


\section{References}

1. Charter IETF MANET WG, Mobile Ad hoc Networking (July 2016)

2. Charter IETF BFD WG, Bidirectional Forwarding Detection (March 2018)

3. Charter IETF BIER WG, Bit Indexed Explicit Replication (March 2018)

4. Charter IETF CCAMP WG, Common Control and Measurement Plane (January 2018)

5. Charter IETF SPRING WG, Source Packet Routing in Networking (October 2018)

6. Charter IETF RAW WG, Reliable and Available Wireless (January 2020)

7. Agarwal, Y., Balaji, B., Gupta, R., Lyles, J., Wei, M., Weng, T.: Occupancydriven energy management for smart building automation. In: ACM Workshop on Embedded Sensing Systems for Energy-Efficiency in Building (BuildSys). pp. 1-6 (2010). https://doi.org/10.1145/1878431.1878433

8. Baytaş, M.A., Yantaç, A.E., Fjeld, M.: Labdesignar: Configuring multicamera motion capture systems in augmented reality. In: Proceedings of the 23rd ACM Symposium on Virtual Reality Software and Technology (2017). https://doi.org/10.1145/3139131.3141778

9. Finn, N., Thubert, P., Varga, B., Farkas, J.: Deterministic Networking Architecture. IETF RFC 8655 (October 2019)

10. Finn, N., Thubert, P.: Deterministic Networking Problem Statement. RFC 8557 (May 2019). https://doi.org/10.17487/RFC8557

11. Grossman, E.: Deterministic Networking Use Cases. IETF RFC 8578 (July 2019)

12. Klemmer, S.R., Ph.D., K.M.E., Landay, J.A.: Integrating physical and digital interactions on walls for fluid design collaboration. Human-Computer Interaction 23(2), 138-213 (2008). https://doi.org/10.1080/07370020802016399

13. Koutsiamanis, R.A., Papadopoulos, G.Z., Jenschke, T.L., Thubert, P., Montavont, N.: Meet the PAREO Functions: Towards Reliable and Available Wireless Networks. In: Proceedings of the IEEE International Conference on Communications (ICC) (2020)

14. Lai, Z., Hu, Y.C., Cui, Y., Sun, L., Dai, N., Lee, H.: Furion: Engineering highquality immersive virtual reality on today's mobile devices. IEEE Transactions on Mobile Computing pp. 1-1 (2019)

15. Lu, Y.: Industry 4.0: A survey on technologies, applications and open research issues. Journal of Industrial Information Integration 6, 1 - 10 (2017). https://doi.org/10.1016/j.jii.2017.04.005

16. Mirsky, G., Chen, M., Black, D.: Operations, Administration and Maintenance (OAM) for Deterministic Networks (DetNet) with IP Data Plane. Internet-Draft draft-mirsky-detnet-ip-oam-02 [work-in-progress], IETF, DetNet WG (March 2020)

17. Papadopoulos, G.Z., Thubert, P., Theoleyre, F., Bernardos, C.: RAW use cases. Internet-Draft draft-bernardos-raw-use-cases-03 [work-in-progress], IETF, RAW WG (March 2020)

18. Papadopoulos, G.Z., Gallais, A., Schreiner, G., Jou, E., Noel, T.: Thorough IoT testbed Characterization: from Proof-of-concept to Repeatable Experimentations. Elsevier Computer Networks 119, 86-101 (2017)

19. Papadopoulos, G.Z., Gallais, A., Schreiner, G., Noel, T.: Importance of Repeatable Setups for Reproducible Experimental Results in IoT. In: Proceedings of the 13th ACM International Symposium on Performance Evaluation of Wireless Ad Hoc, Sensor, and Ubiquitous Networks (PE-WASUN) (2016) 
20. Pavkovic, B., Theoleyre, F., Barthel, D., Duda, A.: Experimental analysis and characterization of a wireless sensor network environment. In: ACM PE-WASUN. pp. 25-32 (2010). https://doi.org/10.1145/1868589.1868595

21. Ratliff, S., Jury, S., Satterwhite, D., Taylor, R., Berry, B.: Dynamic Link Exchange Protocol (DLEP). RFC 8175 (June 2017)

22. Robla-Gómez, S., Becerra, V.M., Llata, J.R., González-Sarabia, E., Torre-Ferrero, C., Pérez-Oria, J.: Working together: A review on safe human-robot collaboration in industrial environments. IEEE Access 5, 26754-26773 (2017)

23. Schnall, S., Hedge, C., Weaver, R.: The immersive virtual environment of the digital fulldome: Considerations of relevant psychological processes. International Journal of Human-Computer Studies 70(8), 561 - 575 (2012). https://doi.org/10.1016/j.ijhcs.2012.04.001

24. Theoleyre, F., Papadopoulos, G.Z., Mirsky, G.: Operations, Administration and Maintenance (OAM) features for RAW. Internet-Draft draft-theoleyre-raw-oamsupport-02 [work-in-progress], IETF, RAW WG (April 2020)

25. Thubert, P.: An Architecture for IPv6 over the TSCH mode of IEEE 802.15.4. Internet-Draft draft-ietf-6tisch-architecture-28 [work-in-progress], IETF, 6TiSCH WG (October 2019)

26. Thubert, P., Cavalcanti, D., Vilajosana, X., Schmitt, C., Farkas, J.: Reliable and Available Wireless Technologies. Internet-Draft draft-thubert-raw-technologies-05 [work-in-progress], IETF, RAW WG (July 2019)

27. Thubert, P., Eckert, T., Brodard, Z., Jiang, H.: BIER-TE extensions for Packet Replication and Elimination Function (PREF) and OAM. Internet-Draft draft-thubert-bier-replication-elimination-03 [work-in-progress], IETF, BIER WG (March 2018)

28. Varga, B., Farkas, J., Berger, L., Malis, A., Bryant, S.: DetNet Data Plane Framework. Internet-Draft draft-ietf-detnet-data-plane-framework-04 [work-in-progress], IETF, DetNet WG (February 2020)

29. Varga, B., Farkas, J., Malis, A.G., Bryant, S.: DetNet Data Plane: IP over IEEE 802.1 Time Sensitive Networking (TSN). Internet-Draft draft-ietf-detnet-ip-overtsn-02, Internet Engineering Task Force (Mar 2020) 